\title{
Comparison of static immersion and intravenous injection systems for exposure of zebrafish embryos to the natural pathogen Edwardsiella tarda
}

\author{
Joost J van Soest, Oliver W Stockhammer, Anita Ordas, Guido V Bloemberg, Herman P Spaink and \\ Annemarie $\mathrm{H}$ Meijer
}

\begin{abstract}
Background: The zebrafish embryo is an important in vivo model to study the host innate immune response towards microbial infection. In most zebrafish infectious disease models, infection is achieved by micro-injection of bacteria into the embryo. Alternatively, Edwardsiella tarda, a natural fish pathogen, has been used to treat embryos by static immersion. In this study we used transcriptome profiling and quantitative RT-PCR to analyze the immune response induced by E. tarda immersion and injection.
\end{abstract}

Results: Mortality rates after static immersion of embryos in E. tarda suspension varied between $25-75 \%$, while intravenous injection of bacteria resulted in 100\% mortality. Quantitative RT-PCR analysis on the level of single embryos showed that expression of the proinflammatory marker genes illb and $\mathrm{mmp} 9$ was induced only in some embryos that were exposed to $E$. tarda in the immersion system, whereas intravenous injection of $E$. tarda led to il1b and mmp9 induction in all embryos. In addition, microarray expression profiles of embryos subjected to immersion or injection showed little overlap. E. tarda-injected embryos displayed strong induction of inflammatory and defense genes and of regulatory genes of the immune response. E. tarda-immersed embryos showed transient induction of the cytochrome P450 gene cypla. This gene was also induced after immersion in Escherichia coli and Pseudomonas aeruginosa suspensions, but, in contrast, was not induced upon intravenous $E$. tarda injection. One of the rare common responses in the immersion and injection systems was induction of irg 11 , a homolog of a murine immunoresponsive gene of unknown function.

Conclusions: Based on the differences in mortality rates between experiments and gene expression profiles of individual embryos we conclude that zebrafish embryos cannot be reproducibly infected by exposure to $E$. tarda in the immersion system. Induction of il1b and mmp9 was consistently observed in embryos that had been systemically infected by intravenous injection, while the early transcriptional induction of cyp $1 a$ and $\mathrm{irg} 1 /$ in the immersion system may reflect an epithelial or other tissue response towards cell membrane or other molecules that are shed or released by bacteria. Our microarray expression data provide a useful reference for future analysis of signal transduction pathways underlying the systemic innate immune response versus those underlying responses to external bacteria and secreted virulence factors and toxins.

\section{Background}

In the last decade the zebrafish has been firmly established as a model for infectious diseases [1-4]. The increasing popularity of the zebrafish is due to its many useful characteristics. The embryos develop fast ex utero and are

\footnotetext{
* Correspondence: a.h.meijer@biology.leidenuniv.nl
Institute of Biology, Leiden University, PO Box 9502, 2300 RA Leiden, The

* Correspondence: a.h.meijer@biology.leidenuniv.nl
Institute of Biology, Leiden University, PO Box 9502, 2300 RA Leiden, The Netherlands
}

(c) 2011 van Soest et al; licensee BioMed Central Ltd. This is an Open Access article distributed under the terms of the Creative

transparent, making it possible to follow infection in vivo. The real-time analysis of infection processes in this model is facilitated by the development of transgenic zebrafish lines with fluorescently marked immune cell populations that can be used in combination with differential fluorescently labeled pathogens [5-8]. In addition, reverse and forward mutagenesis screens are possible, as are antisense knock-down techniques using morpholinos. 
Like all jawed vertebrates the zebrafish possesses an innate and adaptive immune system. Innate immunity forms the first line of defense against invading microorganisms. Humoral components of the innate immune system, such as complement and acute phase proteins, were shown to be expressed in embryos and larvae and could be induced by lipopolysaccharide (LPS) challenge or infection $[9,10]$. The major cell types required for cell-mediated innate immunity, macrophages and neutrophils, also develop during the first days of zebrafish embryogenesis [11-13]. An essential step in innate immunity is the recognition of invading microorganisms by pattern recognition receptor families, the most well studied being the Toll-like receptor (TLR) family. The TLRs activate a signaling pathway leading to a cytokine response and the activation of antimicrobial defense genes [14]. The TLR signaling components are highly conserved between zebrafish and humans $[15,16]$. In adults the innate and adaptive immune systems are tightly connected, however in the zebrafish embryo there is a temporal segregation. Whereas innate immunity is functional as early as 1 day post fertilization (dpf) $[11,17,18]$, adaptive immunity does not reach full maturity until approximately 4 weeks post fertilization $[13,19,20]$. This makes the zebrafish embryo a useful in vivo model to study vertebrate innate immunity separate from adaptive immunity [3].

Bacterial infection models that have been developed in zebrafish differ in mode and time of infection, inoculum size, pathogenicity and host response [2-4]. The most common method of infection is injection, with the caudal vein as injection site at $1 \mathrm{dpf}$ or the yolk circulation valley at $2 \mathrm{dpf}$ [21]. Salmonella typhimurium, a mammalian pathogen, was shown to be lethal to zebrafish embryos after caudal vein injection of a low dose of 25-50 bacteria [22]. In contrast, injection of E. coli or an LPS-mutant of Salmonella typhimurium (Ra-mutant) was not lethal and the bacteria were cleared efficiently by the embryonic innate immune system [22]. Pseudomonas aeruginosa, a broad host range pathogen, capable of infecting plants, invertebrates, and vertebrates, was lethal after injection into the yolk circulation valley at 10-100-fold higher injection inocula than used for S. typhimurium, while Burkholderia cenocepacia was recently shown to cause a lethal infection upon intravenous injection at a dose of less than 10 bacteria [23-25]. At relatively high doses, also gram-positive bacteria such as Streptococcus and Staphylococcus species were shown to be capable of causing lethality upon injection in both adults and embryos [26-29]. Injection of embryos with Mycobacterium marinum does not lead to a lethal infection, but the immune system is unable to clear this bacterium, leading to a chronic infection. This chronic infection is characterized by aggregation of macrophages into granuloma-like structures similar to the tuberculous granulomas found in human tuberculosis patients [17]. The different infection models were useful to study bacterial virulence factors and the response of the host immune system $[3,9,30,31]$.

For experimental screening, intravenous injection of zebrafish embryos is a relatively low throughput method. For high throughput analysis, such as mutant or drug screens, it is highly desirable to have an easier method of infection like static immersion. Thus far, the only bacterial pathogens that were reported to be capable of infecting zebrafish embryos without the need of injection are Edwardsiella tarda and Flavobacterium columnare [32,33], which are Gram-negative naturally occurring fish pathogens. E. tarda is primarily known for infecting channel catfish, Japanese eel and flounder, in which it causes edwarsiellosis, a generalized septicemia. Pressley and colleagues showed that $24 \mathrm{hpf}$ zebrafish embryos immersed for five hours in a suspension of E. tarda had a cumulative mortality rate of $31 \%$ after 14 days, compared to $11 \%$ in the control embryos [32]. In addition, the zebrafish embryos showed peaks in the expression of $\operatorname{tnfa}$ and $i l 1 b$ at 2 and 4 hours post exposure, respectively. In adults, E. tarda is capable of causing infection by static immersion in combination with dermal abrasion [32].

The aim of this study was to compare the robustness of immersion and injection methods for treatment of 1-dayold zebrafish embryos with E. tarda and to identify marker genes that provide a reproducible read-out for the immune response. We set out with a microarray analysis of embryos subjected to immersion in E. tarda, and used E. coli and P. aeruginosa, both non-lethal in the immersion method, for comparison. Several markers were selected for a qPCR time-course analysis of the immersion method and for comparison with caudal vein injection. Marker expression analysis at single embryo level revealed high variation between individuals in response to static immersion. In contrast, qPCR and microarray analysis of single embryos that were systemically infected by caudal vein injection showed a consistent profile of strong activation of the proinflammatory marker genes $i l 1 b$ and mmp9. We conclude that the injection method is best suited for studying the innate immune response towards systemic infection, while the immersion system is useful for studying epithelial or other tissue responses towards cell membrane or other molecules that are shed or released by bacteria.

\section{Results}

\section{Survival of zebrafish embryos after immersion in E. tarda} suspension

In order to test the E. tarda immersion method for future screening applications, we set out to confirm the results obtained by Pressley et al. [32]. To this end, zebrafish embryos at $25 \mathrm{hpf}$ were immersed for $5 \mathrm{~h}$ in $10^{8} \mathrm{CFUs} / \mathrm{ml}$ 
of E. tarda and survival was monitored for four days. The ability of $E$. tarda to cause mortality by static immersion was confirmed, while exposure to heat-killed bacteria did not cause mortality (figure 1). However, the percentage of mortality following E. tarda exposure after 4 days was found to be quite variable, ranging from $25 \%$ to $75 \%$ between different experiments (figure 1). In addition to E. tarda, we also tested the ability of P. aeruginosa to establish a lethal infection by static immersion, using strains PAO1 and PA14. However, even with concentrations up to $10^{9} \mathrm{CFUs} / \mathrm{ml}$, these strains were unable to cause mortality (data not shown).

\section{Microarray analysis of embryos subjected to the immersion system}

The variability of the mortality rate in the E. tarda immersion assay was high. Therefore, we performed microarray analysis on pools of 20 zebrafish embryos immersed at $25 \mathrm{hpf}$ for $5 \mathrm{~h}$ in E. tarda to find markers for a reproducible readout of the immune response as alternative. To determine if we could differentiate between reactions towards pathogenic and non-pathogenic bacteria, $E$. coli $\mathrm{DH} 5 \alpha$ and P. aeruginosa strains PAO1 and PA14 were tested in addition. Surprisingly, E. tarda immersed embryos showed the smallest signature set in terms of gene induction or repression (figure $2 \mathrm{a}$ ). The number of differentially expressed genes after $E$. coli immersion was four times higher (figure $2 \mathrm{~b}$ ), with $P$. aeruginosa PAO1 immersion six times higher (figure 2c), and with $P$. aeruginosa PA14 immersion 13 times higher (figure 2d) (Additional file 1, Table S1).

Surprisingly, very few of the genes up-regulated in the zebrafish embryo after exposure to $E$. tarda were immune related. Although transient induction of $i l 1 b$ and $t n f a$ was

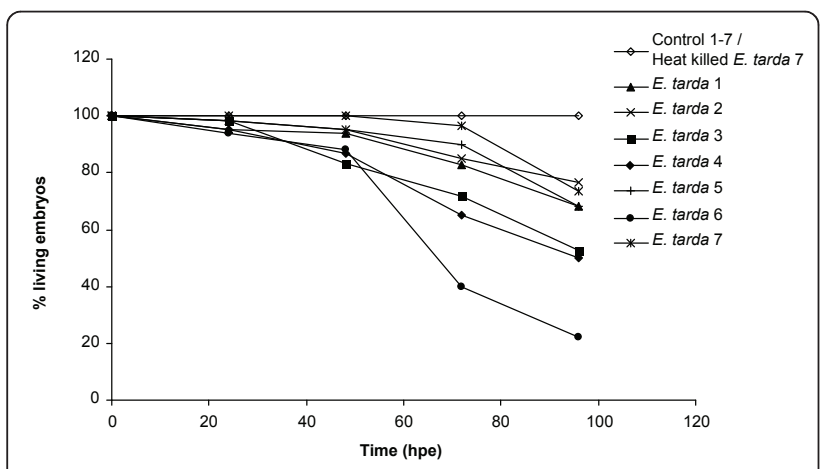

Figure 1 Survival curve of zebrafish embryos treated by immersion in $\boldsymbol{E}$. tarda suspension. For each of the independent 7 experiments 20 embryos at $25 \mathrm{hpf}$ were immersed for $5 \mathrm{~h}$ in $10^{8}$ CFUs/ml of E. tarda or in clean egg water as a control. Subsequently embryos were washed and transferred to fresh egg water, and survival was monitored for 4 days. In one experiment, heat-killed bacteria $\left(45 \mathrm{~min}\right.$ at $95^{\circ} \mathrm{C}$ ) were included as an extra control group. Survival varied between approximately 25 and $75 \%$. previously observed by Pressley et al. [32], no induction of these genes was detected in our microarray analysis. Furthermore, expression of $m m p 9$, one of the most strongly induced markers after Salmonella infection [9], was only slightly up-regulated (1.4 times). In total only 21 genes showed 2-fold or higher levels of up-regulation $(\mathrm{P}<$ 1.0 E-4) after E. tarda exposure (Additional file 1, Table S1). Some of these genes have a possible immune-related function. The highest induced gene after exposure to E. tarda was cypla (9.8-fold induction), which encodes a cytochrome P450 enzyme known to be involved in the toxic response [34,35]. As shown in Additional file 1, Table S1, this gene is also highly induced after $P$. aeruginosa and E. coli exposure. The second highest induced gene was $z g c: 154020$ (6.8-fold), which shows $62.1 \%$ identity with immunoresponsive gene 1 (irg1) from Mus musculus, a gene with homology to bacterial methylcitrate dehydratase, which is up-regulated in murine macrophages after exposure to LPS, cytokines, and mycobacteria [36-39]. Zgc:154020 will hereafter be referred to as irg1-like (irg1l). Like cyp1a, irg1l was also highly up-regulated after $P$. aeruginosa and E. coli exposure. A third gene with a possible immune-related function is stanniocalcin 1 (stc1), which was only induced after E. tarda exposure (2.1-fold). Stanniocalcin is involved in $\mathrm{Ca}^{2+}$ homeostasis in fish [40,41], but in humans has also been implicated in inflammatory responses [42-44].

To compare the responses of zebrafish embryos to immersion with the different bacterial strains, we performed a gene ontology analysis on all genes showing differential expression in the microarray analysis (Additional file 2, Table S2). In embryos immersed in $P$. aeruginosa PAO1 and PA14, and in E. coli, but not in embryos immersed in E. tarda, genes with the GO-term "response to stimulus" were significantly enriched. The largest group of up-regulated genes with this GO-term (61 genes) was observed in the case of immersion with $P$. aeruginosa PA14. Further analysis into the "response to stimulus" GO category revealed that in particular genes with the GO-term "response to stress" were upregulated (41 genes in the case of PA14), while only few genes were associated with the GO-term "immune response" (6 genes in the case of PA14). An overview of the genes with the GO-term "response to stimulus" that were up-regulated in response to the different bacteria is given in Additional file 3, Table S3. The lack of induction of many of the known immune response genes after 5 hours of exposure to E. tarda suggests that at that time, tissue infection has not yet been established.

Time course analysis of marker gene expression in the immersion system

To determine whether a stronger immune response is induced at later time points after exposure to E. tarda, 


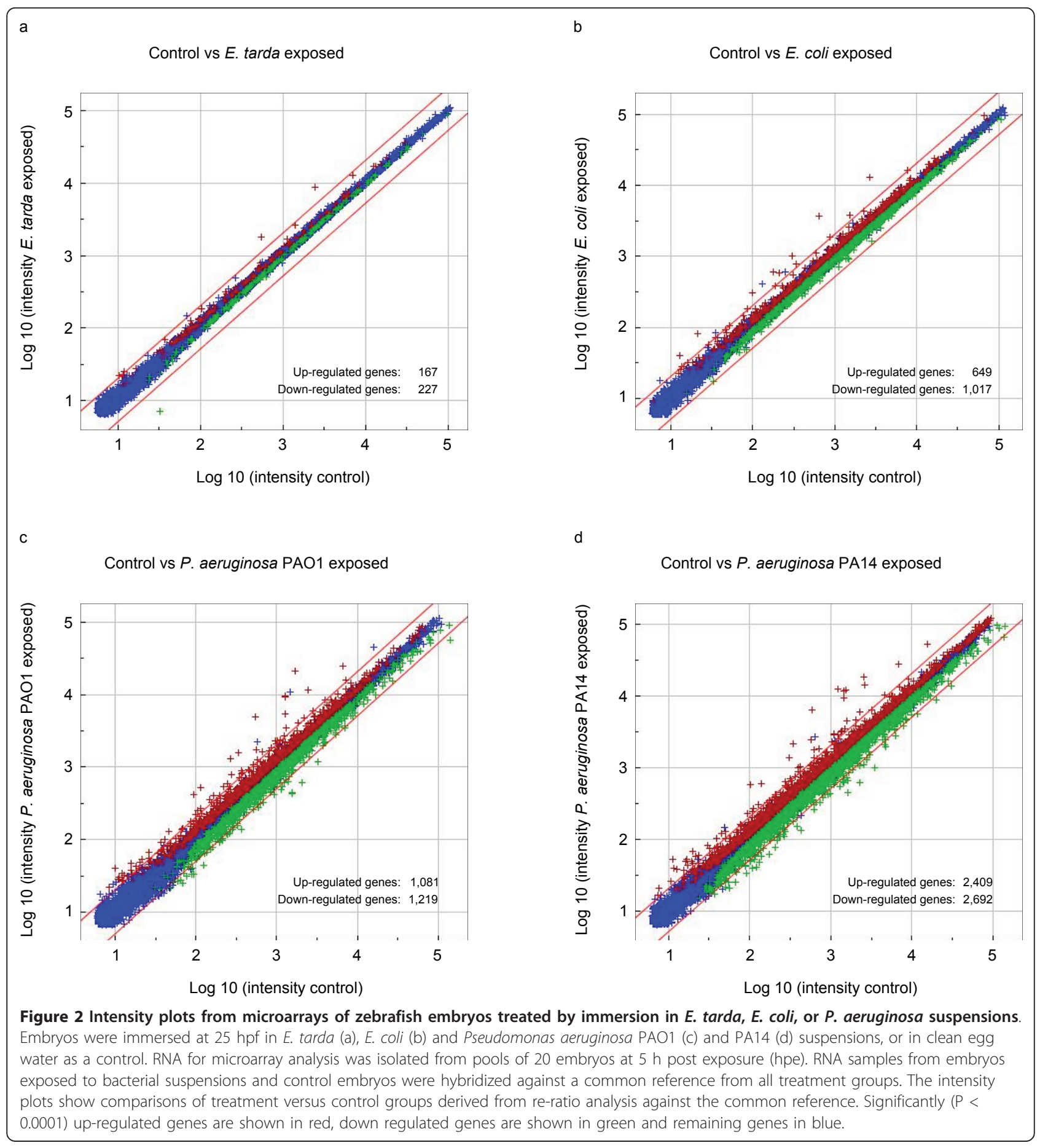

we performed a time-course qPCR analysis of several immune related genes. In addition to the putative immune markers cypla, irg1l and stc1 found in the microarray analysis (Additional file 1, Table S1), the known immune markers $i l 1 b, m m p 9$ and tnfa were chosen for the time course analysis of the E. tarda exposure. Embryos immersed in $10^{8} \mathrm{CFUs} / \mathrm{ml}$ of E. tarda were snap-frozen in pools of 20 embryos at 5,24 and
48 hours post exposure (hpe). RNA was isolated from pools of embryos collected at each time point and the expression of the chosen markers was analyzed. The results showed that cypla is primarily a marker for the early response towards $E$. tarda, showing 10 times higher expression in $E$. tarda-exposed than in untreated embryos at 5 hpe, but less than 3 -fold induction at 24 hpe and no induction at 48 hpe (figure $3 \mathrm{a}$ ). This 


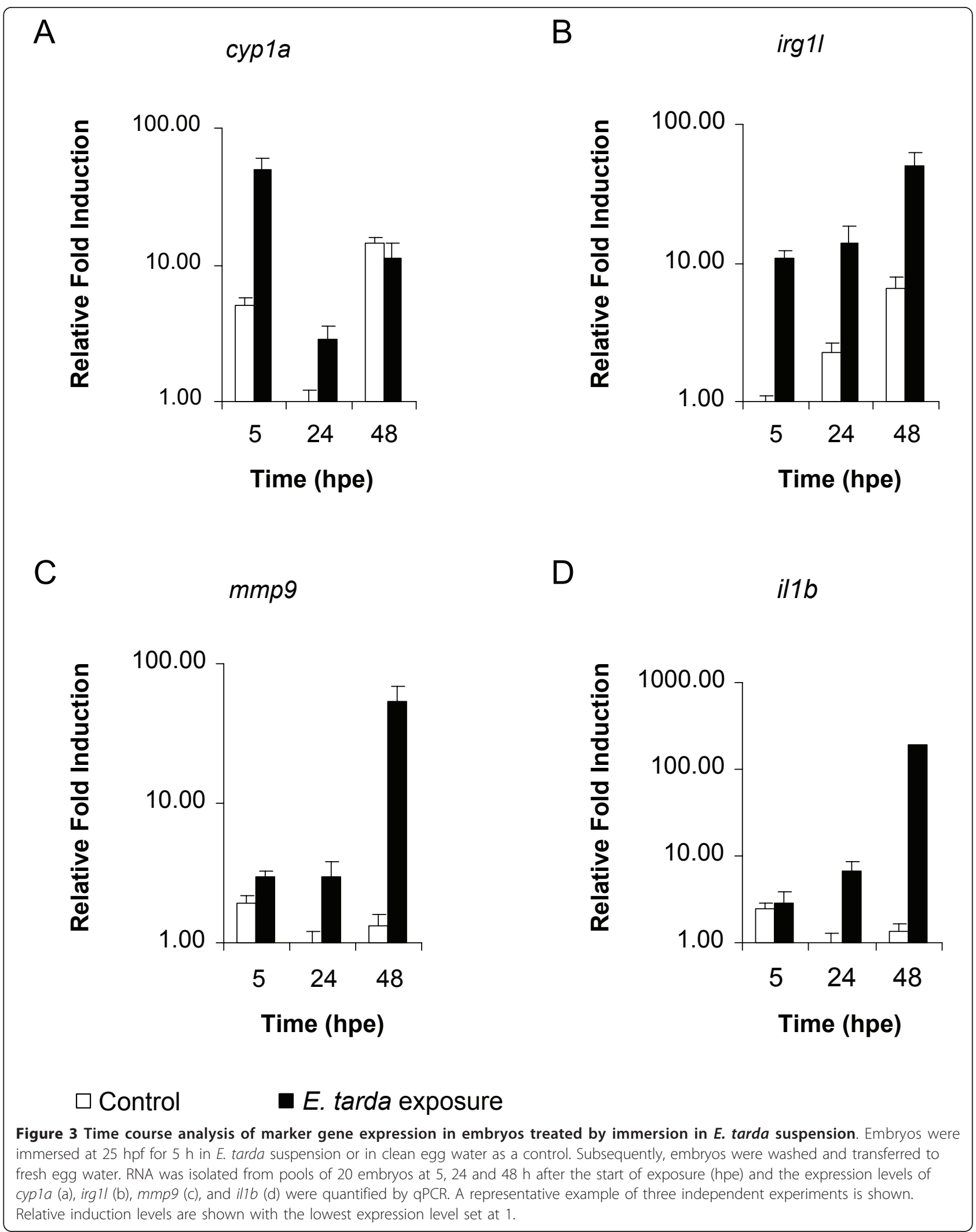


might suggest that cyp1a induction is the result of an epithelial response. Irg1l was induced between 10 and 50 fold at all time points tested (figure $3 \mathrm{~b}$ ). The $m m p 9$ (figure 3c) and $i l 1 b$ genes (figure 3d) showed little to no induction at 5 hpe, but induction started to increase at 24 hpe and reached 54 to 212 -fold induction at $48 \mathrm{hpe}$. The induction of $t n f a$ and stcl was highly variable between the different experiments and therefore excluded in further analyses (data not shown). To test the possibility that the early response in the immersion system might be elicited by cell membrane components or other molecules released by the bacteria, we separated the E. tarda suspension used for the immersion experiments into two fractions by centrifugation. Exposure of embryos either to the wash fluid obtained after centrifugation or to the resuspended bacterial pellet, showed that expression of cypla and irgll was induced to higher levels by the wash fluid than by the washed bacteria, while the opposite was observed for the induction of $i l 1 b$ and mmp9 (Figure 4). Therefore, the early transcriptional induction of cypla and irg1l appears not to be due to bacterial infection.

\section{Immune response in single embryos after static} immersion in E. tarda

The variability in mortality rates in the static immersion system, led us to hypothesize that not all embryos become systemically infected with this method. At 4 days after E. tarda immersion, none of the surviving embryos, even those that were close to dying, showed clear fluorescence
A

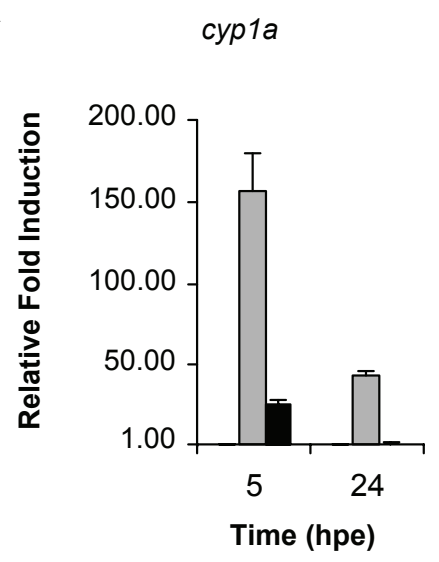

C

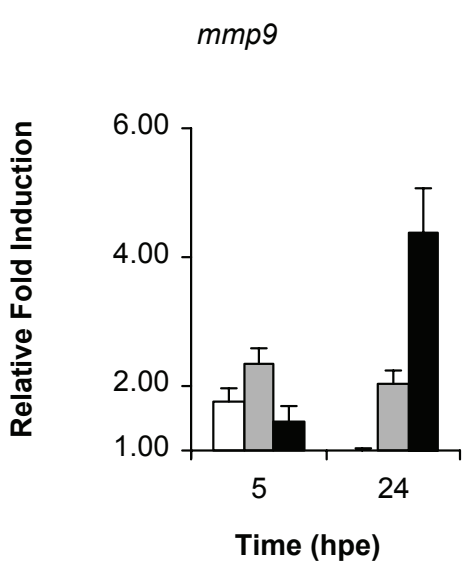

B

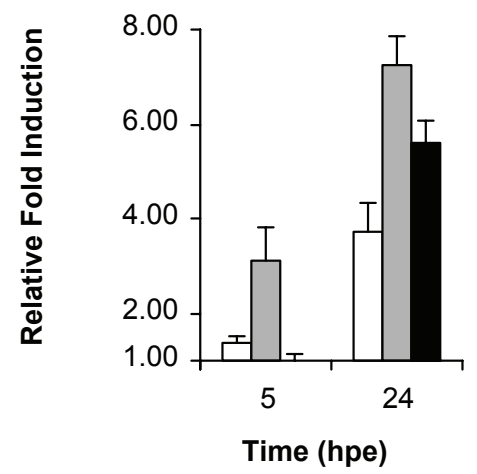

D

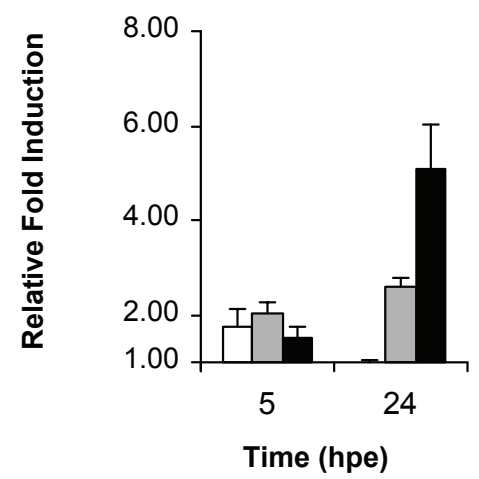

$\square$ Control $\square$ Wash fluid $\square$ Washed E. tarda

Figure 4 Marker gene expression in immersion tests after fractionation of $\boldsymbol{E}$. tarda suspension. The $E$. tarda suspension as used for the immersion experiments in Figure 1-3 was separated into two fractions by centrifugation. Embryos were immersed at $25 \mathrm{hpf}$ for $5 \mathrm{~h}$ in the wash fluid obtained after centrifugation, or in the resuspended bacterial pellet (washed bacteria), or in clean egg water as a control. Subsequently, embryos were washed and transferred to fresh egg water. RNA was isolated from pools of 20 embryos at 5 and $24 \mathrm{~h}$ after the start of exposure (hpe) and the expression levels of cypla (a), irgl/ (b), mmp9 (c), and illb (d) were quantified by qPCR. A representative example of two independent experiments is shown. Relative induction levels are shown with the lowest expression level set at 1. 
of the mCherry marker plasmid. Subsequently, we plated individual surviving embryos for CFU counting. From five surviving embryos, of which three showed a slow heart beat indicative of approaching death, we obtained CFU counts of 140 to 690 per individual embryo. In contrast, the egg water medium of these embryos, kept individually in well plates, contained between 80,000 and 300,000 CFUs. It cannot be ascertained from CFU plating if the surviving embryos were actually infected with low numbers of bacteria or that the low CFU counts resulted from bacteria sticking to the surface epithelium of these embryos. However, it is clear that the surviving embryos did not carry heavy infections.

To further test our hypothesis that not all embryos are systemically infected after immersion, we used an RNAisolation protocol for single embryos [45]. Five single embryos exposed for $5 \mathrm{~h}$ to $10^{8} \mathrm{CFUs} / \mathrm{ml}$ of E. tarda and five single embryos grown under non-inoculated circumstances were snap-frozen at 48 hpe. RNA was isolated from each embryo and qPCR analysis was done on cyp1a, irg1l, mmp9, and il1b (figure 5). Expression of cyp $1 a$ showed little to no induction, similar to what we observed in the analysis of pools of embryos at 48 hpe. The difference in induction of $i l 1 b$ and $m m p 9$ between individual embryos was much more pronounced than we initially expected. Out of the five embryos tested, only one showed a high induction of both markers compared to the control embryos. All embryos showed induction of irg1l, but a strong induction of this gene was only observed in the embryo that showed a high $i l 1 b$ and $m m p 9$ induction, which might indicate that $\mathrm{irg} 1 \mathrm{l}$ is involved in both an initial response to bacterial components and a later systemic immune response.

\section{Immune response in single embryos after caudal vein injection of $E$. tarda}

Results of immersion experiments suggested that induction of $i l 1 b$ and $m m p 9$ expression may be specifically correlated with systemic infection. To exclude that the large variation in $i l 1 b$ and $m m p 9$ induction found after immersion might be due to individual variation in responsiveness of different embryos, we decided to compare the immersion system with intravenous infection. Embryos were injected in the caudal vein with 200 CFUs of E. tarda at $28 \mathrm{hpf}$ and snap-frozen individually at 4 and 8 hours post infection (hpi) after which RNA was isolated. As before, qPCR analysis was done on cypla, irg1l, mmp9, and $i l 1 b$ (figure 6). The results show that the genes irgll, $m m p 9$, and $i l 1 b$ were induced at much higher levels than in the immersion system, whereas cypla showed similar induction (2-5-fold) as in the immersion system in some embryos or no induction in other embryos. Expression of $i l 1 b$ was clearly induced in all embryos at $4 \mathrm{hpi}$, while $m m p 9$ was induced only in two embryos at this time point and irg1l was not induced. Although induction of $m m p 9$ and irg $1 l$ at 8 hpi was consistent, the induction levels showed large variation, ranging between 7 - and 180-fold for $m m p 9$ and between 4- and 140-fold for irg1l. Induction levels of $i l 1 b$ between individual embryos were the least variable, ranging between 5- and 50-fold at 4 hpi and between 10 - and 30 -fold at $8 \mathrm{hpi}$. Compared to injection of 200 CFUs, injection of 25 CFUs resulted in lower $i l 1 b$ and $m m p 9$ induction levels (Additional file 4, Figure S1). Furthermore, these genes were induced at much higher levels by 200 CFUs of live bacteria than by the same dose of heat-killed bacteria.

In addition to the analysis of cyp $1 a, \operatorname{irg} 1 l, m m p 9$ and $i l 1 b$ induction, we monitored the embryos for two days after injection for appearance of fluorescence from the mCherry-labeled E. tarda and for survival. In all injection experiments embryos showed fluorescence at 24 hpi (data not shown) and mortality after injection was very consistent, reaching $100 \%$ at 48 hpi (figure 7). Based on these results we conclude that reproducible systemic infection of zebrafish embryos can be achieved by microinjection of $E$. tarda bacteria, accompanied by induction of $i l 1 b$ and $m m p 9$ expression.

\section{Microarray analysis of embryos infected by caudal vein injection}

Microarray analysis was used to further characterize the immune response in response to microinjection of E. tarda bacteria and compare this with the previous microarray results of the immersion system and with our published data of the response to Salmonella typhimurium injection [9]. Single infected and mock-injected embryos were analyzed at $8 \mathrm{hpi}$ in triplicate. In gene ontology analysis we observed significant enrichment of the GO-terms "immune system process" and "response to stimulus" (Additional file 5, Table S4), whereas these GO-terms were not enriched in results of the immersion method (Additional file 2, Table S2). In addition, functional annotation using DAVID [46] showed significant enrichment of the KEGG pathways for apoptosis and for Toll-like receptor, adipocytokine, NOD-like receptor, insulin, MAP kinase, RIG-I-like receptor, ErbB, and JakStat signaling. Manual annotation of the induced gene group showed several representatives of the categories complement activation and acute phase response, immune-related transcription factors and signaling components, cytokines and chemokines, apoptosis, and defense response (Additional file 6, Figure S2). In addition, many genes that were not previously linked to the immune response were differentially expressed, including genes involved in signal transduction, transporting activity and metabolism (Additional file 6, Figure S2). Out of 498 significantly regulated probes at $8 \mathrm{hpi}$ (Additional file 7 , Table S5), only 2 down-regulated probes (for $v$ tg6 and 


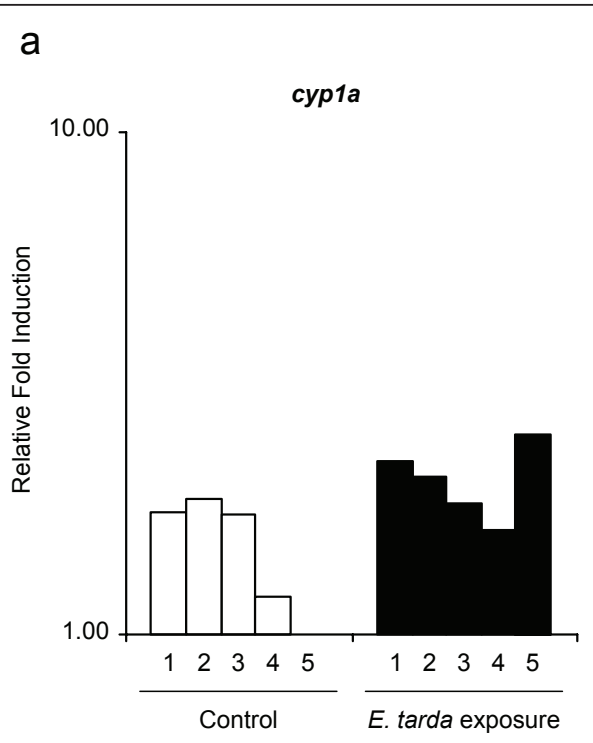

C

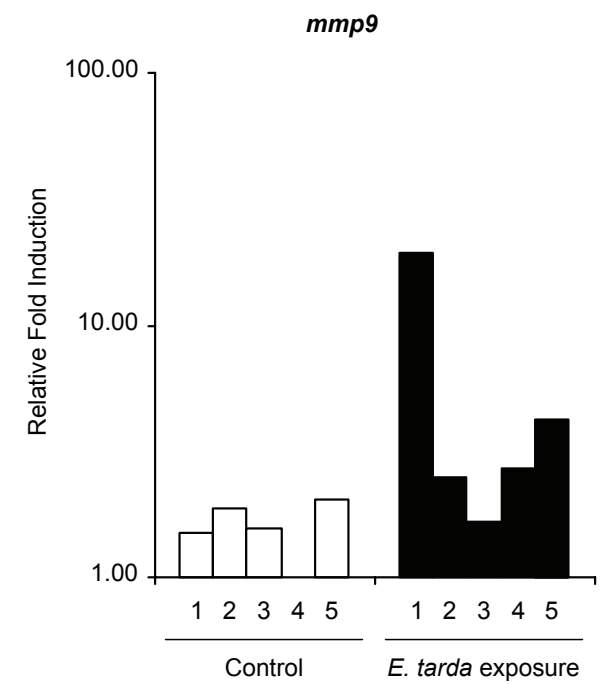

b

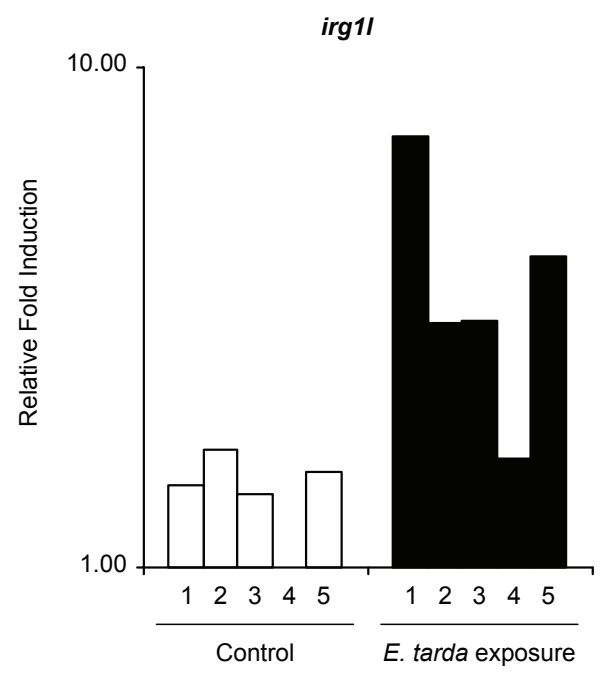

d

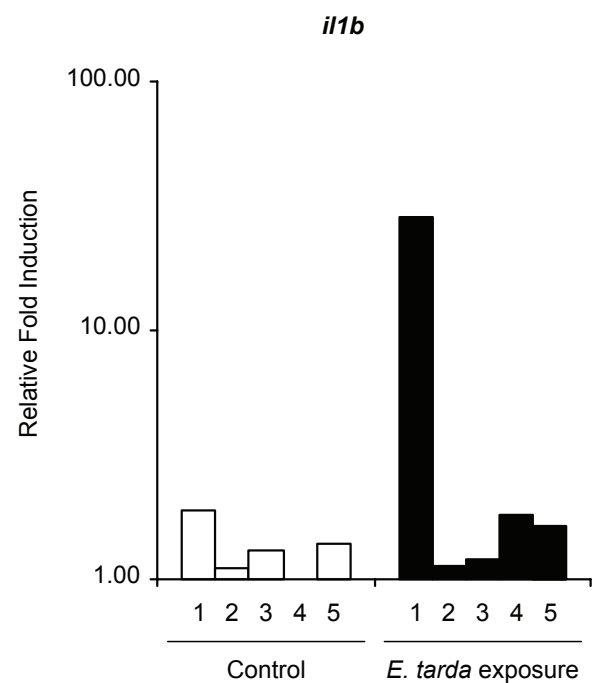

Figure 5 Marker gene expression in individual embryos treated by immersion in E. tarda suspension. Pools of 20 embryos were immersed at $25 \mathrm{hpf}$ for $5 \mathrm{~h}$ in E. tarda suspension or in clean egg water as a control. Subsequently, embryos were washed and transferred to fresh egg water. RNA was isolated from 5 single embryos at $48 \mathrm{~h}$ after the start of exposure (hpe) and expression levels of cyp 1a (a), irg 1/ (b), mmp9 (c) and illb (d) were measured by qPCR. Relative induction levels are shown with the lowest expression level set at 1.

an unannotated transcribed locus) and 1 up-regulated probe (for an unknown gene) were also significantly changed in the immersion system at 5 hpe. The microarray comparison supports that the transcriptional signatures of embryos subjected to immersion and injection are markedly different, although it should be noted that the immersion and injection data are not directly comparable due to a few hours difference in time to respond to the bacteria and in the developmental stage of assessment. The E. tarda injection microarray data were also compared with our previous microarray data set of intravenous Salmonella typhimurium infection of embryos at 2, 5 and 8 hpi [9]. This comparison showed an overlap of 141 probes with significantly changed expression in response to both pathogens (Additional file 7, Table S5). These probes represented among others $t n f b, i l 1 b, c x c l$ c1c, mmp 9, ncf1, mxc, pglyrp5, hamp1 and several signal transduction (e.g. tlr5b, irak3, nfkbiaa, pim1, socs $1 / 3 a$ / $3 b$ ) and transcription factor genes (e.g. atf3, elf3, fos, junb, irg9/11, rel, stat1) (Additional file 7, Table S5). 




\section{Discussion}

Zebrafish is being established as an alternative vertebrate model to murine models for infection research. To enable large scale mutant and chemical screening the development of an easily applicable infection test system is highly desired. In this report we studied the effectiveness and variability of treatment of zebrafish embryos by static immersion in Edwardsiella tarda, a method previously described by Pressley et al. [32], in comparison with the caudal vein injection method.

In order to perform large scale screenings, a model test system should be optimized for a reproducible response. Our results confirmed the ability of E. tarda to cause mortality in zebrafish embryos after static immersion. However, the mortality rate was highly variable between different experiments, ranging from 25 $75 \%$, comparable to the mortality rate of $31 \%$ reported by Pressley et al [32]. In order to find a more reproducible readout, we performed microarray analysis on zebrafish embryos that had been exposed to $E$. tarda by static immersion. Surprisingly, only a small number of genes showed differential expression. In contrast, a much larger number of genes were regulated by immersion in bacterial suspensions of $E$. coli and $P$. aeruginosa strains 


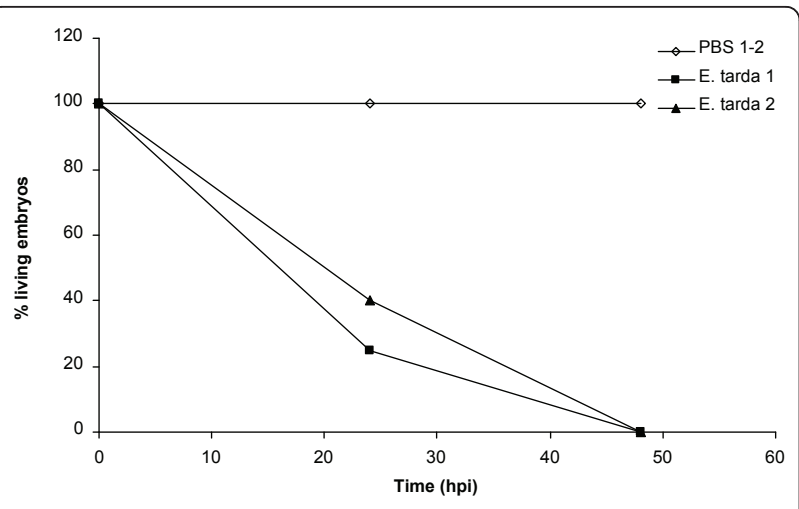

Figure 7 Survival curve of embryos infected by injection of E. tarda. Survival after injection of approximately 200 CFUs of $E$. tarda into the caudal vein of embryos at $28 \mathrm{hpf}$ was monitored for two days, after which no embryos survived. Control embryos were injected with PBS. Experiments were performed in duplicate. In each experiment 20 embryos were used per treatment group.

PAO1 and PA14 that do not cause any mortality. In addition, very few immune-related genes were induced by immersion in $E$. tarda and we observed no induction of $i l 1 b$ and $t n f a$, which showed transient induction patterns between 2 and $12 \mathrm{hpi}$ in the study of Pressley et al. [32].

Interestingly, cyp $1 a$ was highly induced by all tested bacteria. In E. tarda immersion experiments the induction of this gene preceded that of $i l 1 b$ and $m m p 9$ induction. Our results suggest that this gene is not induced by direct exposure to the bacteria, but by released cell membrane components or other molecules. Expression of cyp $1 a$ was most strongly induced by $P$. aeruginosa. Cypla is known to be induced by toxic chemicals in vascular endothelium, but also in the epithelium of the gills [34,35]. P. aeruginosa PAO1 and PA14 are known to secrete large amounts of toxins and protein virulence factors [47-50]. Since cyp1a belongs to the cytochrome P450 family, its induction might be involved in a detoxification response. The observation that many of the genes regulated by $P$. aeruginosa are associated with the GO term "response to stress", and the lack of enrichment of genes with the GO-term "immune system process" is consistent with a response to toxins rather than an immune response to systemic infection.

A further time-course analysis by qPCR of pools of embryos subjected to $E$. tarda immersion showed strong induction of $i l 1 b$ and $m m p 9$ after 48 hours. In addition, the irg1l gene, one of the few immune-related genes identified in the microarray study, was also induced at later time points after exposure to E. tarda. The irg1l gene is homologous to mammalian irg1, expression of which in murine macrophages is induced by cytokines, agonists of TLR signalling, and by mycobacterial infections [36-39]. Sequence similarity of $\operatorname{irg} 1 \mathrm{ll}$ and mammalian irg1 with bacterial methylcitrate dehydratases suggests an important role in metabolism, but the function in vertebrates remains unknown. When we analyzed gene expression at the level of single embryos we observed that $i l 1 b$ and $m m p 9$ were highly expressed in only one out of five treated embryos. Expression of $\mathrm{irg} 1 \mathrm{l}$ was induced in all embryos, but only at a high level in the same embryos that also showed induction of $i l 1 b$ and $m m p 9$. One possible explanation for the variable results of the static immersion assay is that embryos can individually differ in their resistance towards $E$. tarda. To test this, we compared the immersion system with intravenous injection of bacteria. In contrast to the relatively low and highly variable mortality rates that we observed with the immersion method, injection of bacteria resulted in a reproducible rate of $100 \%$ mortality within 2 days. Strong individual differences in levels of gene expression were also observed in the injection system, but nevertheless, induction of the proinflammatory marker genes $i l 1 b$ and $m m p 9$ was positive in all embryos and their induction levels correlated with the dose of live bacteria injected. Furthermore, microarray experiments with single injected embryos showed a consistent profile of strong activation of proinflammatory and defense genes and regulatory genes of the immune response. The observed gene expression profiles are concordant with those observed for intravenous Salmonella typhimurium infection of embryos at similar time periods after injection [9]. Detailed comparisons of the responses to $E$. tarda and S. typhimurium infections will be part of a follow-up study that will also address the function of essential immune regulators in these models.

Since only a subset of embryos in the immersion assay showed induction of immune response markers and mortality it is conceivable that only these embryos were systemically invaded by $E$. tarda bacteria or that nonresponsive embryos were invaded by a much lower number of bacteria. Neither fluorescence monitoring nor CFU plating indicated that embryos become heavily infected close before dying. On the contrary, bacteria were present in high abundance in the egg water medium and only few were associated with dying embryos. It therefore remains uncertain whether infection or toxic insult is the actual cause of mortality in the immersion system. It is possible that the variable immune gene inductions and mortality rates resulted from slight epithelial damage to embryos that occurred during dechorionating and washing procedures, providing sites of entry for bacteria. Instead of exposure at $1 \mathrm{dpf}$, we used the same immersion protocol on embryos of $3 \mathrm{dpf}$, which is the developmental stage when the mouth opens and the gut begins to be colonized by environmental bacteria [51]. We followed survival until $5 \mathrm{dpf}$, which is the time-point up to which larvae do not fall under the European animal experimentation law, but 
did not observe mortality within that time (data not shown).

Besides being more practical for high-throughput screening, an immersion system might be preferred as a more natural route of infection compared to injection methods. However, we conclude that the E. tarda immersion method as applied here on 1-day-old zebrafish embryos is not suitable to achieve reproducible systemic infection. Therefore, unless a more virulent strain can be identified, injection remains the preferred method of infection for screening purposes. On the other hand, the immersion system is shown to be useful for studying epithelial or other tissue responses towards cell membrane or other molecules that are shed or released by bacteria. An alternative solution for high-throughput screening of systemic infection is the use of robotic yolk injection system recently developed for Mycobacterium marinum infection [52]. However, the wild type E. tarda FL6-60 strain used here causes early lethality after yolk injection (data not shown). The use of less virulent (wild type or mutant) strains might provide a solution for this problem. In any case, our gene expression profiling data sets will be necessary for comparisons to the immune response in such alternative yolk infection methods.

\section{Conclusions}

Zebrafish embryos proved to be remarkably resistant to becoming systemically infected after immersion in bacterial suspensions of $E$. tarda, whereas they are strongly susceptible to intravenous injection of this pathogen. While the microarray expression profile of intravenously infected embryos indicates a strong inflammatory response, the transcriptional signature of embryos subjected to immersion was markedly different. Our data suggest that most of the early transcriptional responses in the immersion system may reflect an epithelial or other tissue response towards cell membrane or other molecules that are shed or released by bacteria. Therefore, our studies on the expression analysis in the bacterial immersion system will be useful for future analysis of signal transduction pathways underlying responses to external bacteria and secreted putative virulence factors and toxins. Transient induction of the cytochrome P450 gene cypla was specifically observed in immersion experiments but not when embryos were systemically infected by injection. In addition, our identification of the $\mathrm{irg} 1 \mathrm{l}$ gene as a rapid response factor to externally added bacteria deserves further study of the underlying signal transduction pathway as compared to systemic tissue responses. Although irgll is also up-regulated during systemic infection, its expression kinetics in embryos immersed in $E$. tarda is very different from that of well-known inflammation genes such as $i l 1 b$ and $m m p 9$. Considering the important function of epithelial cells in cross talk with cells of the innate immune system, as recently underscored by studies in zebrafish [31], further analysis of infection modes using the identified marker genes will help to better understand the systemic response of tissues toward an infection in a whole organism context.

\section{Methods}

\section{Zebrafish husbandry}

Zebrafish were handled in compliance with the local animal welfare regulations and maintained according to standard protocols (zfin.org). An albino strain was used for all immersion and injection experiment, except for the microarray study of injected embryos that was performed with wild type zebrafish. Embryos were grown at $28.5-30^{\circ} \mathrm{C}$ in egg water $(60 \mu \mathrm{g} / \mathrm{ml}$ Instant Ocean salts). For the duration of bacterial injections embryos were kept under anesthesia in egg water containing $0.02 \%$ buffered 3 -aminobezoic acid ethyl ester (tricaine; Sigma-Aldrich).

\section{Bacterial immersion and injection experiments}

Edwardsiella tarda strain FL6-60 obtained from Dr. P. Klesius (USDA, Auburn, AL) is the identical strain as used in the study of Pressley et al. [32]. Identity of this strain was confirmed by performing nucleotide sequencing of the entire genome using Illumina technology with a 180-fold coverage (Genbank accessions CP002154 and CP002155). FL6-60 was grown over night on tryptic soy agar (Difco) at $28^{\circ} \mathrm{C}$ and subsequently a liquid culture in tryptic soy broth (TSB, Difco) was inoculated and grown overnight at $28^{\circ} \mathrm{C}$ with shaking at $150 \mathrm{rpm}$. Pseudomonas aeruginosa PAO1 and PA14 and Escherichia coli were grown over night in Luria-Bertani broth (LB) [53] at $37^{\circ} \mathrm{C}$. For immersion experiments bacterial cultures were centrifuged in $50 \mathrm{ml}$ tubes and the pellet was subsequently suspended in egg water to a final $10^{8} \mathrm{CFU} / \mathrm{ml}$ for E. tarda and E. coli, and $10^{9} \mathrm{CFU} / \mathrm{ml}$ for P. aeruginosa. Embryos were dechorionated at $24 \mathrm{hpf}$ by a 3-5 min pronase treatment $\left(2 \mathrm{mg} / \mathrm{ml}\right.$ in embryo medium prewarmed to $\left.30^{\circ} \mathrm{C}\right)$ and left to recover for one hour in egg water. Subsequently pools of 20 embryos in 6-well plates were immersed in $5 \mathrm{ml}$ of the bacterial suspension and incubated for 5 hours at $28^{\circ} \mathrm{C}$. After 5 hours of incubation, the embryos were either snap-frozen in liquid nitrogen or transferred to a new 6-wells plate, washed 3 times in egg water, and incubated at $28^{\circ} \mathrm{C}$ in $5 \mathrm{ml}$ of egg-water. For CFU plating experiments, embryos were kept individually in $2.5 \mathrm{ml}$ of egg water in 24-well plates.

For caudal vein injection experiments, E. tarda labeled with mCherry [54] was washed and subsequently suspended in PBS (phosphate-buffered saline) to a final $10^{8}$ $\mathrm{CFU} / \mathrm{ml}$. Embryos were manually dechorionated at $24 \mathrm{hpf}$. Approximately 200 CFUs of $E$. tarda were injected into the blood island after the onset of blood flow at $28 \mathrm{hpf}$, or PBS was injected as a control. After injection, embryos 
were kept at $28^{\circ} \mathrm{C}$ and snap-frozen in liquid nitrogen at the required time points.

\section{RNA isolation from pools of embryos}

Pools of 20 - 30 embryos were snap-frozen in liquid nitrogen and subsequently stored at $-80^{\circ} \mathrm{C}$. Embryos were homogenized in $1 \mathrm{ml}$ of TRI reagent (Ambion), and subsequently total RNA was extracted according to the manufacturer's instructions. The RNA samples were incubated for $20 \mathrm{~min}$ at $37^{\circ} \mathrm{C}$ with $10 \mathrm{U}$ of DNaseI (Roche Applied Science) to remove residual genomic DNA before purification using the RNeasy MinElute Cleanup kit (Qiagen) according to the RNA clean-up protocol. The integrity of the RNA was confirmed by lab-on-chip analysis using the 2100 Bioanalyzer (Agilent Technologies). Samples used for microarray analysis had an average RNA integrity number value of 9 and a minimum RNA integrity number value of 8 .

\section{RNA isolation from single embryos}

The single embryo RNA isolation procedure was performed according to de Jong et al. [45]. Embryos were individually snap-frozen in liquid nitrogen and subsequently stored at $-80^{\circ} \mathrm{C}$. A frozen embryo was crushed with a chilled pestle and homogenized in $300 \mu \mathrm{l}$ of TRI reagent (Ambion). $60 \mu \mathrm{l}$ of chloroform was added and the mixture was transferred to a $1.5 \mathrm{ml}$ reaction tube containing $50 \mathrm{mg}$ phase lock gel (Eppendorf) and incubated at room temperature for 5 minutes. The mixture was centrifuged at $12000 \mathrm{~g}$ at $4^{\circ} \mathrm{C}$ for 15 minutes, after which the aqueous phase was transferred to a fresh tube. 1 volume of $70 \%$ ethanol was added and the mixture transferred to a RNeasy MinElute Cleanup kit (Qiagen) column which was centrifuged 15 seconds at 8000 g. $500 \mu \mathrm{l}$ RPE buffer from the kit was applied to the column and centrifuged $15 \mathrm{sec}-$ onds at 8000 g. $500 \mu \mathrm{l} 80 \%$ ethanol was applied to the column and centrifuged 2 minutes at $8000 \mathrm{~g}$. The collection tube was replaced and the column centrifuged 5 minutes at 14000 g. $14 \mu \mathrm{H}_{2} \mathrm{O}$ was applied to the column and centrifuged 1 minute at $14000 \mathrm{~g}$. The average amount of RNA isolated from a single embryo was $500 \mathrm{ng}$.

\section{Microarray analysis}

The microarray slides were custom-designed by Agilent Technologies as previously described [9]. The slides contained in total 43,371 probes of a 60 -oligonucleotide length.

Amino-allyl-modified amplified RNA (aRNA) was synthesized in one amplification round from total RNA using the Amino Allyl MessageAmp II aRNA Amplification kit (Ambion). The amount of total RNA used was $1 \mu \mathrm{g}$ in experiments using RNA from pooled embryos and $400 \mathrm{ng}$ in experiments using RNA from single embryos. Subsequently, $6 \mu \mathrm{g}$ of amino-allyl-modified aRNA was used for coupling of monoreactive Cy3 and Cy5 dyes (GE Healthcare) and column purified. Samples from embryos immersed in E. tarda, E. coli, or P. aeruginosa suspensions or untreated control embryos were labeled with $\mathrm{Cy} 5$ and hybridized against a Cy3-labeled common reference that consisted of a mixture of all samples from the immersion experiments. E. tarda and control immersions were analyzed in triplicate using pools of 20 embryos and compared with single experiments of E. coli, $P$. aeruginosa PAO1 and P. aeruginosa PA14 immersion. For the E. tarda injection study, infected embryos and control embryos injected with the PVP-carrier solution were labeled with Cy5 and analyzed in triplicate against a Cy3labeled common reference. Dual-color hybridization of the microarray chips was performed at ServiceXS according to Agilent protocol G4140-90050 version 5.7 (http://www. Agilent.com) for two-color microarray-based gene expression analysis.

Microarray data were processed from raw data image files with Feature Extraction Software 9.5.3 (Agilent Technologies). Processed data were subsequently imported into Rosetta Resolver 7.0 (Rosetta Biosoftware) and subjected to default ratio error modeling. The raw data were submitted to the Gene Expression Omnibus database (http://www.ncbi.nlm.nih.gov/geo) under accession no. GSE28486. To compare samples from treatment groups to the control samples re-ratio analyses were performed using the Rosetta built-in re-ratio with common reference application. Data were analyzed at the level of UniGene clusters (UniGene build no. 105) and at probe level. Significance cut-offs for the ratios were set at 1.5fold change at $P<10^{-4}$ for analysis at UniGene cluster level and $P<10^{-5}$ for analysis at probe level.

Gene ontology (GO) analysis was performed using the GeneTools eGOn v2.0 web-based gene ontology analysis software (http://www.genetools.microarray.ntnu.no) [55]. KEGG pathway analysis was performed using DAVID tools for functional annotation (http://david.abcc.ncifcrf. gov/) [46]. In addition, genes were manually annotated based on information in the ZFIN (zfin.org) and NCBI Entrez Gene databases, and PubMed abstracts.

\section{CDNA synthesis and quantitative reverse transcriptase PCR}

For RNA samples from pooled embryos, cDNA synthesis reactions were performed in a $20 \mu \mathrm{l}$ mixture of $500 \mathrm{ng}$ of RNA, $4 \mu \mathrm{l}$ of $5 \mathrm{x}$ iScript reaction mix (Bio-Rad Laboratories), and $1 \mu \mathrm{l}$ of iScript reverse transcriptase (Bio-Rad Laboratories). For RNA samples from single embryos, cDNA synthesis reactions were performed in a $10 \mu \mathrm{lmix}$ ture of $100 \mathrm{ng}$ of RNA, $2 \mu \mathrm{l}$ of $5 \mathrm{x}$ iScript reaction mix (Bio-Rad Laboratories), and $0.5 \mu \mathrm{l}$ of iScript reverse transcriptase (Bio-Rad Laboratories). The reaction mixtures 
were incubated at $25^{\circ} \mathrm{C}$ for $5 \mathrm{~min}, 42^{\circ} \mathrm{C}$ for $30 \mathrm{~min}$, and $85^{\circ} \mathrm{C}$ for $5 \mathrm{~min}$.

Real-time PCR was performed using the Chromo4 Real-time PCR detection system (Bio-Rad Laboratories) according to the manufacturer's instructions. Each reaction was performed in a $25-\mu \mathrm{l}$ volume comprised of $1 \mu \mathrm{l}$ of cDNA, $12.5 \mu \mathrm{l}$ of $2 \mathrm{x}$ iQ SYBR Green Supermix (BioRad Laboratories), and 10 pmol of each primer. Cycling parameters were $95^{\circ} \mathrm{C}$ for $3 \mathrm{~min}$ to activate the polymerase, followed by 40 cycles of $95^{\circ} \mathrm{C}$ for $15 \mathrm{~s}$ and $59^{\circ} \mathrm{C}$ for $45 \mathrm{~s}$. Fluorescence measurements were taken at the end of each cycle. Melting curve analysis was performed to verify that no primer dimers were amplified. All reactions were performed as technical duplicates. For normalization, peptidylprolyl isomerase A-like (ppial), which showed no changes over the infection time course series, was taken as reference. Results were analyzed using the $\Delta \Delta C_{t}$ method. Sequences of forward and reverse primers are described in Additional file 8, Table S6.

\section{Additional material}

Additional file 1: Genes up-regulated in zebrafish embryos at $5 \mathrm{~h}$ after immersion in E. tarda, E. coli, $P$. aeruginosa PAO1, and $P$. aeruginosa PA14. The table lists the genes that had a significant $(\mathrm{P}<$ $0.0001)$ signature at $5 \mathrm{~h}$ after immersion of zebrafish embryos at $25 \mathrm{hpf}$ in E. tarda, E. coli, P. aeruginosa PAO1 or P. aeruginosa PA14 suspension.

Additional file 2: Gene ontology analysis of the up-regulated genes in zebrafish embryos after immersion in E. tarda, E. coli, $P$. aeruginosa PA01, and $P$. aeruginosa PA14. Genes up-regulated significantly ( $p<0.0001$, no fold change cut-off) after immersion in $E$. tarda, E. coli, P. aeruginosa PAO1, and P. aeruginosa PA14 were subjected to a master-target statistical test using the web-based eGOn software. The table indicates the number of genes associated with the indicated GO categories for the master (i.e. all genes on the microarray) and targets (i.e. the differentially expressed gene lists of the treatment groups). Yellow indicates significant enrichment of GO-categories in the treatment groups, and blue indicates significant underrepresentation ( $P<$ 0.05)

Additional file 3: Up-regulated genes with the GO-term "response to stimulus" after immersion in E. tarda, E. coli, P. aeruginosa PAO1, and $P$. aeruginosa PA14. Gene ontology analysis using eGOn identified $5,22,41$ and 61 genes (indicated with + ) that were up-regulated $(P<$ 0.0001) after immersion of zebrafish embryos in E. tarda, E. coli, P. aeruginosa PAO1, or P. aeruginosa PA14, and that were associated with the GO-term 'response to stimulus'.

Additional file 4: Marker gene expression in individual embryos in response to injection of different doses of live and heat-killed E. tarda. Expression levels of $m m p 9$ (a) and il1b (b) were measured by qPCR in single embryos at $8 \mathrm{~h}$ after injection (hpi) of approximately 25 or 200 CFUs of live or heat-killed $\left(45 \mathrm{~min}\right.$ at $95^{\circ} \mathrm{C}$ ) E. tarda into the caudal vein embryos at $28 \mathrm{hpf}$. Control embryos were injected with PBS. Relative induction levels are shown with the lowest expression level set at 1. Lines with * indicate a significant difference of $P<0.05$ (tested by one-way ANOVA analysis with the Bonferroni method as post-hoc test).

Additional file 5: Gene ontology analysis of the up-regulated genes in zebrafish embryos injected with $E$. tarda. Genes up-regulated significantly up-regulated $(P<0.00001$ ) after injection of zebrafish embryos with $E$. tarda were subjected to a master-target statistical test using the web-based eGOn software. The table indicates the number of genes associated with the indicated GO categories for the master (i.e. all genes on the microarray) and the target (i.e. differentially expressed genes after E. tarda injection). Yellow indicates significant enrichment of GO-categories in the treatment groups, and blue indicates significant underrepresentation $(P<0.05)$

Additional file 6: Heat-map and annotations of genes differentially expressed at $\mathbf{8} \mathbf{h}$ after injection of $E$. tarda. Genes were manually annotated and assigned to functional groups based on GO annotations of the zebrafish genes and their human homologues and on searching of PubMed abstracts. (a) Genes previously implicated to be involved in the immune response or novel genes with strong sequence similarity to those genes, (b) genes with known or predicted functions not previously linked to the immune response. Up-regulation is indicated by

increasingly bright shades of yellow and down-regulation by increasingly bright shades of blue. The significance cut-off for the analysis was set at $P<0.00001$.

Additional file 7: Genes showing differential expression in zebrafish embryos at $\mathbf{8} \mathbf{h}$ after injection of $\boldsymbol{E}$. tarda. The table lists the probes that had a significant $(P<0.00001)$ signature at $8 \mathrm{~h}$ after injection of 200 CFU of E. tarda into the caudal vein of zebrafish embryos at $28 \mathrm{hpf}$. Genes were manually annotated and assigned to functional groups based on GO annotations of the zebrafish genes and their human homologues and on searching of PubMed abstracts. Genes are divided into 3 categories: 1: annotated genes previously implicated in the vertebrate immune response and novel/hypothetical genes with sequence similarity to these immune response genes; 2 : annotated or novel/hypothetical genes whose known or predicted functions have not been linked to the vertebrate immune response; 3: genes with unknown function. Genes from categories 1 and 2 are ordered by functional annotation groups. For comparison fold change and P-values are shown of probes that also had a significant signature in the E. tarda immersion microarray or in previous microarray data of S. typhimurium injection at 2 , 5 and 8 hpi [9].

Additional file 8: qPCR primer sequences. Primer sequences and Genbank accessions for genes analyzed in this study.

\section{Acknowledgements}

We thank Dr. Philip Klesius (USDA, Auburn, AL) for providing us with E. tarda strain FL6-60, Roel de Haan for help with GPCR, and Davy de Wit and Ulrike Nehrdich for fish care. This work was supported by the European Commission $6^{\text {th }}$ framework project ZF-TOOLS (LSHG-CT-2006-037220).

\section{Authors' contributions}

JJVS designed and conducted experiments, analyzed the data, and wrote the manuscript. OWS and AO contributed to the experiments and data analysis. GVB, HPS and AHM conceived and supervised the study, and edited the manuscript. All authors read and approved the final version.

\section{Competing interests}

The authors declare that they have no competing interests.

Received: 9 May 2011 Accepted: 17 October 2011 Published: 17 October 2011

\section{References}

1. Kanther M, Rawls JF: Host-microbe interactions in the developing zebrafish. Curr Opin Immunol 2010, 22:10-19.

2. Meeker ND, Trede NS: Immunology and zebrafish: Spawning new models of human disease. Dev Comp Immunol 2008, 32:745-757.

3. Meijer AH, Spaink HP: Host-pathogen interactions made transparent with the zebrafish model. Curr Drug Targets 2011, 12:1000-1007.

4. Sullivan C, Kim CH: Zebrafish as a model for infectious disease and immune function. Fish Shellfish Immunol 2008, 25:341-350.

5. Hall C, Flores MV, Crosier K, Crosier P: Live cell imaging of zebrafish leukocytes. Methods Mol Biol 2009, 546:255-271.

6. Meijer AH, van der Sar AM, Cunha C, Lamers GEM, Laplante MA, Kikuta H, Bitter W, Becker TS, Spaink HP: Identification and real-time imaging of a myc-expressing neutrophil population involved in inflammation and 
mycobacterial granuloma formation in zebrafish. Dev Comp Immunol 2008, 32:36-49.

7. Renshaw SA, Loynes CA, Trushell DMI, Elworthy S, Ingham PW, Whyte MKB: A transgenic zebrafish model of neutrophilic inflammation. Blood 2006, 108:3976-3978.

8. Ellett F, Pase L, Hayman JW, Andrianopoulos A, Lieschke GJ: mpeg1 promoter transgenes direct macrophage-lineage expression in zebrafish. Blood 2011, 117:e49-56.

9. Stockhammer OW, Zakrzewska A, Hegedus Z, Spaink HP, Meijer AH: Transcriptome profiling and functional analyses of the zebrafish embryonic innate immune response to Salmonella infection. J Immunol 2009, 182:5641-5653.

10. Wang Z, Zhang S, Wang G: Response of complement expression to challenge with lipopolysaccharide in embryos/larvae of zebrafish Danio rerio: Acquisition of immunocompetent complement. Fish Shellfish Immunol 2008, 25:264-270.

11. Herbomel $P$, Thisse $B$, Thisse C: Ontogeny and behaviour of early macrophages in the zebrafish embryo. Development 1999, 126:3735-3745.

12. Le Guyader D, Redd MJ, Colucci-Guyon E, Murayama E, Kissa K, Briolat V, Mordelet E, Zapata A, Shinomiya H, Herbomel P: Origins and unconventional behavior of neutrophils in developing zebrafish. Blood 2008, 111:132-141.

13. Willett CE, Cortes A, Zuasti A, Zapata AG: Early hematopoiesis and developing lymphoid organs in the zebrafish. Dev Dyn 1999, 214:323-336.

14. Mogensen TH: Pathogen recognition and inflammatory signaling in innate immune defenses. Clin Microbiol Rev 2009, 22:240-273.

15. Meijer AH, Gabby Krens SF, Medina Rodriguez IA, He S, Bitter W, Ewa SnaarJagalska B, Spaink HP: Expression analysis of the Toll-like receptor and TIR domain adaptor families of zebrafish. Mol Immunol 2004, 40:773-783.

16. Stein C, Caccamo M, Laird G, Leptin M: Conservation and divergence of gene families encoding components of innate immune response systems in zebrafish. Genome Biol 2007, 8:R251.

17. Davis JM, Clay H, Lewis JL, Ghori N, Herbomel P, Ramakrishnan L: Real-time visualization of Mycobacterium-macrophage interactions leading to initiation of granuloma formation in zebrafish embryos. Immunity 2002, 17:693-702.

18. Herbomel P, Thisse B, Thisse C: Zebrafish early macrophages colonize cephalic mesenchyme and developing brain, retina, and epidermis through a M-CSF receptor-dependent invasive process. Dev Biol 2001, 238:274-88.

19. Davidson AJ, Zon LI: The 'definitive' (and 'primitive') guide to zebrafish hematopoiesis. Oncogene 2004, 23:7233-7246.

20. Lam SH, Chua HL, Gong Z, Lam TJ, Sin YM: Development and maturation of the immune system in zebrafish, Danio rerio: a gene expression profiling, in situ hybridization and immunological study. Dev Comp Immunol 2004, 28:9-28.

21. Cui C, Benard EL, Kanwal Z, Stockhammer OW, Van der Vaart M, Zakrzewska A, Spaink HP, Meijer AH: Infectious disease modeling and innate immune function in zebrafish. Methods Cell Biol 2011, 105:273-308.

22. van der Sar AM, Musters RJP, van Eeden FJM, Appelmelk BJ, Vandenbroucke-Grauls CMJE, Bitter W: Zebrafish embryos as a model host for the real time analysis of Salmonella typhimurium infections. Cell Microbiol 2003, 5:601-611.

23. Brannon MK, Davis JM, Mathias JR, Hall CJ, Emerson JC, Crosier PS, Huttenlocher A, Ramakrishnan L, Moskowitz SM: Pseudomonas aeruginosa Type III secretion system interacts with phagocytes to modulate systemic infection of zebrafish embryos. Cell Microbiol 2009, 11:755-768.

24. Clatworthy AE, Lee JS-W, Leibman M, Kostun Z, Davidson AJ, Hung DT: Pseudomonas aeruginosa infection of zebrafish involves both host and pathogen determinants. Infect Immun 2009, 77:1293-1303.

25. Vergunst AC, Meijer AH, Renshaw SA, O'Callaghan D: Burkholderia cenocepacia creates an intra-macrophage replication niche in zebrafish embryos, followed by bacterial dissemination and establishment of systemic infection. Infect Immun 2010, 78:1495-1508.

26. Lin A, Loughman JA, Zinselmeyer BH, Miller MJ, Caparon MG: Streptolysin S inhibits neutrophil recruitment during the early stages of Streptococcus pyogenes Infection. Infect Immun 2009, 77:5190-5201.

27. Lin B, Chen S, Cao Z, Lin Y, Mo D, Zhang H, Gu J, Dong M, Liu Z, Xu A: Acute phase response in zebrafish upon Aeromonas salmonicida and Staphylococcus aureus infection: Striking similarities and obvious differences with mammals. Mol Immunol 2007, 44:295-301.
28. Neely MN, Pfeifer JD, Caparon M: Streptococcus-zebrafish model of bacterial pathogenesis. Infect Immun 2002, 70:3904-3914.

29. Prajsnar TK, Cunliffe VT, Foster SJ, Renshaw SA: A novel vertebrate model of Staphylococcus aureus infection reveals phagocyte-dependent resistance of zebrafish to non-host specialized pathogens. Cell Microbiol 2008, 10:2312-2325.

30. Phelps HA, Neely MN: Evolution of the zebrafish model: from development to immunity and infectious disease. Zebrafish 2005, 2:87-103.

31. Volkman HE, Pozos TC, Zheng J, Davis JM, Rawls JF, Ramakrishnan L: Tuberculous granuloma induction via interaction of a bacterial secreted protein with host epithelium. Science 2010, 327:466-469.

32. Pressley ME, Phelan PE III, Witten PE, Mellon MT, Kim CH: Pathogenesis and inflammatory response to Edwardsiella tarda infection in the zebrafish. Dev Comp Immunol 2005, 29:501-513.

33. Chang MX, Nie P: RNAi suppression of zebrafish peptidoglycan recognition protein 6 (zfPGRP6) mediated differentially expressed genes involved in Toll-like receptor signaling pathway and caused increased susceptibility to Flavobacterium columnare. Vet Immunol Immunopathol 2008, 124:295-301.

34. Guiney PD, Smolowitz RM, Peterson RE, Stegeman JJ: Correlation of 2,3,7,8tetrachlorodibenzo-p-dioxin induction of cytochrome P4501A in vascular endothelium with toxicity in early life stages of lake trout. Toxicol Appl Pharmacol 1997, 143:256-273.

35. Jönsson ME, Brunström B, Brandt I: The zebrafish gill model: Induction of CYP1A, EROD and PAH adduct formation. Aquat Toxicol 2009, 91:62-70.

36. Basler T, Jeckstadt S, Valentin-Weigand P, Goethe R: Mycobacterium paratuberculosis, Mycobacterium smegmatis, and lipopolysaccharide induce different transcriptional and post-transcriptional regulation of the IRG1 gene in murine macrophages. J Leukoc Biol 2006, 79:628-638.

37. Degrandi $D$, Hoffmann $R$, Beuter-Gunia C, Pfeffer $K$ : The proinflammatory cytokine-induced IRG1 protein associates with mitochondria. J Interferon Cytokine Res 2009, 29:55-68.

38. Lee CG, Jenkins NA, Gilbert DJ, Copeland NG, O'Brien WE: Cloning and analysis of gene regulation of a novel LPS-inducible CDNA. Immunogenetics 1995, 41:263-270.

39. Shi S, Nathan C, Schnappinger D, Drenkow Jr, Fuortes M, Block E, Ding A, Gingeras TR, Schoolnik G, Akira S, et al: MyD88 primes macrophages for full-scale activation by Interferon-gamma yet mediates few responses to Mycobacterium tuberculosis. J Exp Med 2003, 198:987-997.

40. Tseng DY, Chou MY, Tseng YC, Hsiao CD, Huang CJ, Kaneko T, Hwang PP: Effects of stanniocalcin 1 on calcium uptake in zebrafish (Danio rerio) embryo. Am J Physiol Regul Integr Comp Physiol 2009, 296:R549-557.

41. Wagner GF, Dimattia GE, Davie JR, Copp DH, Friesen HG: Molecular cloning and CDNA sequence analysis of coho salmon stanniocalcin. Mol Cell Endocrinol 1992, 90:7-15.

42. Chakraborty A, Brooks H, Zhang P, Smith W, McReynolds MR, Hoying JB, Bick R, Truong L, Poindexter B, Lan H, Elbjeirami W, Sheikh-Hamad D: Stanniocalcin-1 regulates endothelial gene expression and modulates transendothelial migration of leukocytes. Am J Physiol Renal Physiol 2007, 292:F895-904.

43. Kanellis J, Bick R, Garcia G, Truong L, Tsao CC, Etemadmoghadam D, Poindexter B, Feng L, Johnson RJ, Sheikh-Hamad D: Stanniocalcin-1, an inhibitor of macrophage chemotaxis and chemokinesis. Am J Physiol Renal Physiol 2004, 286:F356-F362.

44. Wang Y, Huang L, Abdelrahim M, Cai Q, Truong A, Bick R, Poindexter B, Sheikh-Hamad D: Stanniocalcin-1 suppresses superoxide generation in macrophages through induction of mitochondrial UCP2.J Leukoc Biol 2009, 86:981-988.

45. de Jong M, Rauwerda H, Bruning O, Verkooijen J, Spaink H, Breit T: RNA isolation method for single embryo transcriptome analysis in zebrafish. BMC Res Notes 2010, 3:73.

46. Dennis $\mathrm{G} \mathrm{Jr}$, Sherman BT, Hosack DA, Yang J, Gao W, Lane HC, Lempicki RA DAVID: database for annotation, visualization, and integrated discovery. Genome Biol 2003, 4:P3.

47. Darby C, Cosma CL, Thomas JH, Manoil C: Lethal paralysis of Caenorhabditis elegans by Pseudomonas aeruginosa. Proc Natl Acad Sci USA 1999, 96:15202-15207.

48. Galloway DR: Pseudomonas aeruginosa elastase and elastolysis revisited: recent developments. Mol Microbiol 1991, 5:2315-2321. 
49. Peters JE, Galloway DR: Purification and characterization of an active fragment of the LasA protein from Pseudomonas aeruginosa: enhancement of elastase activity. J Bacteriol 1990, 172:2236-2240.

50. Wick MJ, Frank DW, Storey DG, Iglewski BH: Structure, function, and regulation of Pseudomonas Aeruginosa exotoxin A. Ann Rev Microbiol 1990, 44:335-363.

51. Rawls JF, Mahowald MA, Goodman AL, Trent CM, Gordon Jl: In vivo imaging and genetic analysis link bacterial motility and symbiosis in the zebrafish gut. Proc Natl Acad Sci USA 2007, 104:7622-7627.

52. Carvalho R, de Sonneville J, Stockhammer OW, Savage NDL, Veneman WJ, Ottenhoff THM, Dirks RP, Meijer AH, Spaink HP: A high-throughput screen for tuberculosis progression. PLOS ONE 2011, 6:e16779.

53. Sambrook JaDR: Molecular Cloning: A Laboratory Manual Cold Spring Harbor NY, USA: Cold Spring Laboratory Press; 2001.

54. Lagendijk EL, Validov S, Lamers GEM, Weert Sd, Bloemberg GV: Genetic tools for tagging Gram-negative bacteria with mCherry for visualization in vitro and in natural habitats, biofilm and pathogenicity studies. FEMS Microbiol Lett 2010, 305:81-90

55. Beisvag V, Junge F, Bergum H, Jolsum L, Lydersen S, Gunther CC, Ramampiaro H, Langaas M, Sandvik A, Laegreid A: GeneTools - application for functional annotation and statistical hypothesis testing. BMC Bioinformatics 2006, 7:470.

doi:10.1186/1471-2172-12-58

Cite this article as: van Soest et al: Comparison of static immersion and intravenous injection systems for exposure of zebrafish embryos to the natural pathogen Edwardsiella tarda. BMC Immunology 2011 12:58.

\section{Submit your next manuscript to BioMed Central and take full advantage of:}

- Convenient online submission

- Thorough peer review

- No space constraints or color figure charges

- Immediate publication on acceptance

- Inclusion in PubMed, CAS, Scopus and Google Scholar

- Research which is freely available for redistribution

Submit your manuscript at www.biomedcentral.com/submit 